\title{
The effect of aerobic dance intervention on brain spontaneous activity in older adults with mild cognitive impairment: A resting-state functional MRI study
}

\author{
MING QI $^{1 *}$, YI ZHU $^{2 *}$, LING ZHANG $^{1 *}$, TING WU $^{3}$ and JIE WANG ${ }^{1}$ \\ Departments of ${ }^{1}$ Radiology, ${ }^{2}$ Rehabilitation and ${ }^{3}$ Neurology, \\ The First Affiliated Hospital of Nanjing Medical University, Nanjing, Jiangsu 210029, P.R. China
}

Received June 4, 2018; Accepted November 14, 2018

DOI: $10.3892 /$ etm.2018.7006

\begin{abstract}
The current study aimed to evaluate the effect of a specially designed moderate-intensity aerobic dance (SDMIAD) on brain spontaneous activity in older adults with mild cognitive impairment (MCI) using resting-state functional magnetic resonance imaging (RS-fMRI). A total of 38 subjects with MCI were recruited to the current study and were randomized into two groups: Exercise $(E G, n=19)$ and control $(\mathrm{CG}, \mathrm{n}=19)$. The EG was treated with a SDMIAD and usual care for 3 months. The $\mathrm{CG}$ only received usual care. None of the patients were administered medicine that affected cognition during the intervention. The cognitive assessments and RS-fMRI examination were performed on the two groups at recruitment and after 3 months. The cognitive functions were assessed by various neuropsychological tests. The brain spontaneous activity change was assessed using an index, the amplitude of low-frequency fluctuation (ALFF) of fMRI signal. Cognitive assessments demonstrated that EG had significantly improved results in the Mini-Mental State Examination, the Montreal Cognitive Assessment, Wechsler Memory Scale-Revised Logical Memory (WMS-R LM) and the Symbol Digit Modalities Test (all $\mathrm{P}<0.05$ ), and the difference in changes in WMS-R LM from baseline to 3 months between the EG and the CG was also statistically significant $(\mathrm{P}<0.05)$ after 3 months of SDMIAD. The performance of all the cognitive assessments did not demonstrate significant differences in CG. Compared with baseline, EG exhibited significantly increased ALFF in several areas, including the
\end{abstract}

Correspondence to: Professor Jie Wang, Department of Radiology, The First Affiliated Hospital of Nanjing Medical University, 300 Guangzhou Road, Nanjing, Jiangsu 210029, P.R. China

E-mail: jiewang33@126.com

${ }^{*}$ Contributed equally

Key words: mild cognitive impairment, aerobic dance, resting-state functional magnetic resonance imaging, amplitude of low-frequency fluctuation bilateral fronto-temporal, entorhinal, anterior cingulate and parohippocampal cortex after 3 months of SDMIAD $(\mathrm{P}<0.05)$; whereas the CG exhibited significantly increased ALFF only in a few areas, including right temporal and posterior cingulate cortex $(\mathrm{P}<0.05)$. The SDMIAD may effectively improve the cognitive function in older adults with MCI. RS-fMRI provided a quantitative method for evaluating the effect of aerobic exercise on cognitive function.

\section{Introduction}

Mild cognitive impairment (MCI) has been proposed as a symptom in prodromal Alzheimer's disease (AD) (1). MCI is associated with early memory loss, ineffective neural processing and increased risk of $\mathrm{AD}$, which is the most common neurodegenerative disease in the elderly that causes the decline of memory, language and other cognitive functions (2). Epidemiologically, AD affects $\sim 5 \%$ of individuals $>65$ years of age and $20 \%$ of those $>80$ years of age, with the rate of prevalence doubling every 5 years (3). An increasing number studies have been investigating the potential risk factors for AD (4-6). In particular, physical inactivity has been regarded as a marked risk factor for cognitive decline (7) and cognitive impairments, including AD and MCI $(4,8)$.

Physical activity, such as aerobic exercise, is beneficial to physical and mental disorders (9). A study suggested that aerobic exercise was effective at preventing or delaying the development of AD (10). Studies using objectively measured aerobic exercise have revealed that the intensity rather than the amount of exercise was associated with cognitive performance $(11,12)$. A recent study revealed that moderately intense aerobic exercise serves an important part in maintaining brain health (13). The potential protective mechanism of aerobic exercise on patients with AD and MCI remains unclear.

Resting-state functional magnetic resonance imaging (RS-fMRI) is a noninvasive technique and has provided new opportunities to explore the changes of the structure and function in the brain (14). The blood oxygen level dependent low-frequency oscillation signal in the RS-fMRI denotes spontaneous activity of neurons (15). The average square root of low-frequency bands $(0.01-0.08 \mathrm{~Hz})$ at each voxel is taken as the amplitude of low-frequency fluctuation (ALFF), 
which is assumed to reflect the intensity of brain spontaneous activity $(16,17)$. Several studies have demonstrated that exercise intervention will increase gray and white matter volume in the prefrontal cortex (18), improve the resting-state functional connectivity between the medial prefrontal cortex and medial temporal lobe (19) and increase the function of the dorsolateral prefrontal, posterior parietal and anterior cingulate cortex in the executive control network $(20,21)$. Previous studies have assessed the brain activity of patients with MCI using ALFF analysis without any exercise intervention (22-24). In the current study, the authors took advantage of the ALFF as a supplementary method of cognitive function assessments to evaluate the effects of a specially designed moderate-intensity aerobic dance (SDMIAD) on older adults with MCI and determine the effect of SDMIAD patients with MCI.

\section{Materials and methods}

Subjects. A total of 50 volunteers (17 males, 33 females) with MCI aged 50-85 years old were recruited in the current study. They were recruited from the dementia clinic of the First Affiliated Hospital of Nanjing Medical University (Nanjing, China) or the local community through newspaper advertisement in June 2014. Following screening, only 38 participants were enrolled in the present study and were randomly allocated into two groups: Exercise $(E G, n=19)$ or control (CG, $\mathrm{n}=19$ ). None of the patients were administered medicine that affected cognition during the intervention, including donepezil and memantine. The designer of the current study and all participants were blind to the group assignment. A flowchart demonstrating the process of initial recruitment to study completion is presented in Fig. 1.

Subjects included in the current study met the following criteria: i) Aged 50-85 years old; ii) diagnosed with MCI according to the National Institute on Aging and Alzheimer's Association guidelines (25); iii) a history of memory impairment lasting $\geq 3$ months (26); iv) a Mini-Mental State Examination (MMSE) score of 25-30 (27); v) a Montreal Cognitive Assessment (MoCA; Beijing version) score of $\leq 26$ (28); vi) no medical history of cerebrovascular disease, Parkinson disease or depression; vii) not participating in other trials. Subjects excluded from the current study met the following criteria: i) Diagnosed with vascular dementia using the National Institute of Neurological Disorders and Stroke and the Association Internationale pour la Recherché et l'Enseignement en Neurosciences criteria (29); ii) a Hachinski Ischemic score of $>4$ (26) and iii) could not take the cognitive function and MRI tests due to disorders, including deafness, blindness or severe language disorders.

\section{Exercise intervention}

i) SDMIAD. Subjects in the EG were taught the new dance routine for 2 weeks, when they had learn the dance and could follow the routine correctly. The majority of the subjects danced three times a week together for 3 months. The new dance routine was designed by a physical therapist who had $>5$ years of physical therapy experience. The SDMIAD lasted $35 \mathrm{~min}$, including a 5-min warm-up, a 25-min dance with a target heart rate and a 5-min cool-down to music. The warm-up consisted of four movements: Stepping and breathing, head movements, side bending, and turning. The dance routines consisted of eight movements: Knee bending, heel lifts, boxing, shoulder movements, kicking, square stepping, sculling and jumping. The cool-down consisted of three movements: Shoulder movements, stepping and breathing, and low-speed stepping. The dance routine was deemed to be quite complicated as it contained repetition and variety; therefore it required memory, concentration and dual-task function to complete the dance correctly. During the dance, there were two physical therapists monitoring the heart rate with a cardiotachometer to ensure that all subjects maintain a pre-arranged intensity level during training. One patient in the EG discontinued the intervention due to illness, thus they were excluded from the current study.

ii) The intensity of the training. The American College of Sports Medicine recommended that the intensity of aerobic training should be $55-95 \%$ of the maximum heart rate $\left(\mathrm{HR}_{\max }\right)$ or $40-85 \%$ of the maximum heart rate reserve $(30)$. The authors selected an $\mathrm{HR}_{\max }$ of $60-80 \%$ as the target heart rate during the aerobic dance, which is considered moderately. All subjects were involved in a 'symptom-limited maximal exercise test' (31) prior to inclusion in the current study and the $\mathrm{HR}_{\max }$ was measured during the test. The target heart rate was then calculated.

Neuropsychological tests. A comprehensive neuropsychological test battery for cognitive function was performed at baseline and after the 3-month intervention. Multiple domains of cognition including general cognition, memory and executive function were assessed. Also, the balance control was evaluated. All the neuropsychological tests were completed by the same neuropsychologist, who was blind to the group assignment: i) MMSE is the most commonly used screening tool for dementia. Scores range between 0 and 30, with higher scores indicating better cognitive function. A cut-off of 24 (following education correction) is recommended for cognitive disorders; ii) MoCA Beijing version is a screening tool for MCI and mild dementia, and has been revealed to have high sensitivity and specificity for differentiating individuals with MCI from healthy adults in several developed countries and areas (32). Scores range from 0 to 30, with higher scores indicating better cognitive function. A cut-off of 26 (following education correction) is recommended for cognitive disorders and 16 for patients with AD in the United States (33); iii) the Wechsler Memory Scale-Revised Logical Memory (WMS-R LM) is mainly used to assess logical memory (34). During the test, the examiner read aloud two short stories to the participant, each with 25 content units. In the present study, the stories from the Japanese version of the WMS-R LM test were translated into Chinese. Following each story, the examiner asked the participant to repeat the story immediately as close to verbatim as possible; iv) Digit Span Test-forward (DST-F; Chinese version) and DST-backward (B; Chinese version) are commonly used to evaluate immediate memory and attention (35). During each test, the examiner asked the subject to recall the numbers in a forward order (DST-F) or backward order (DST-B), which were presented at the rate of one every second; v) trail Making Test Part A and B (TMT-A, B) are usually used to evaluated executive function (36). The TMT 


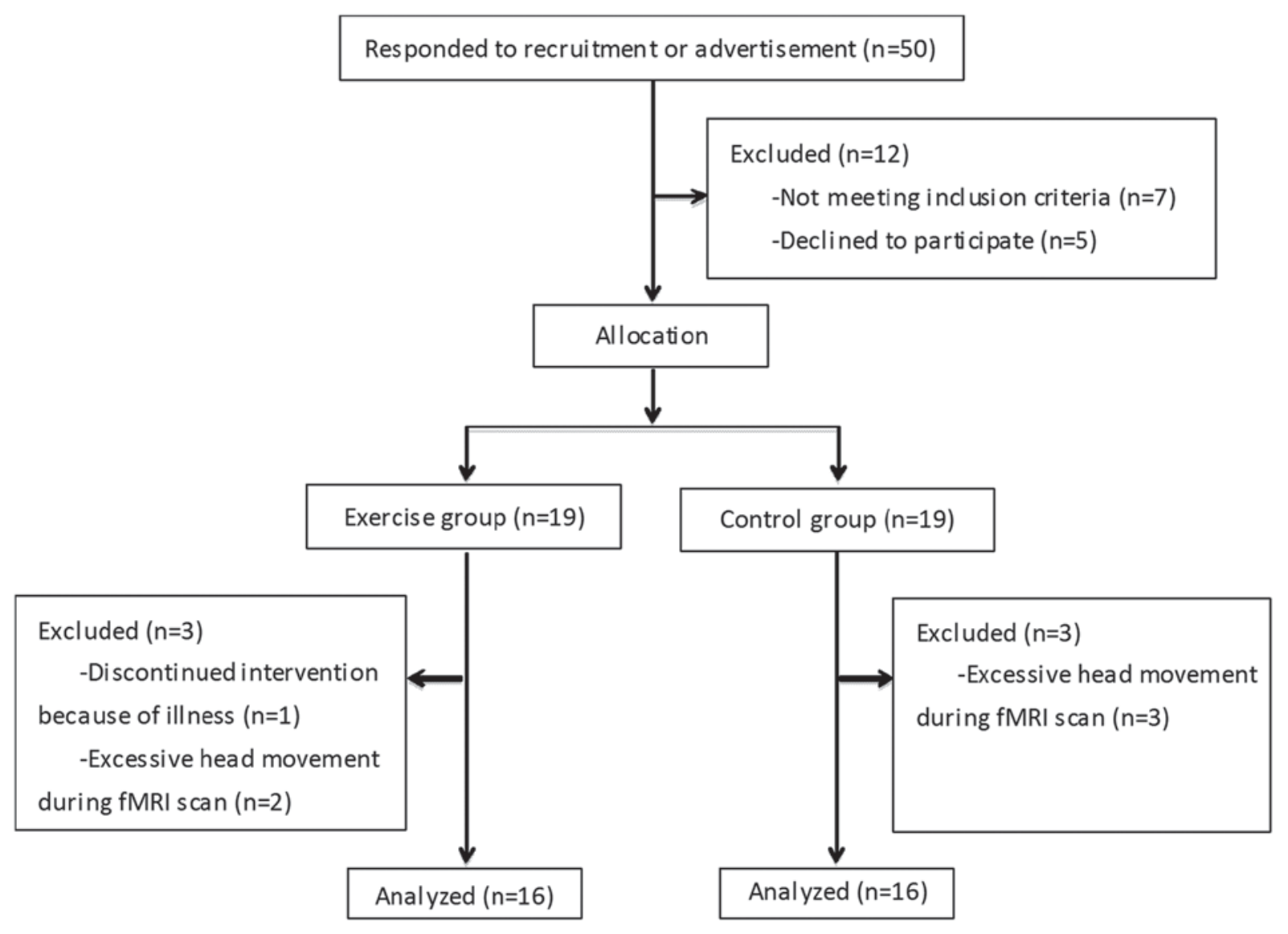

Figure 1. A flow chart of the recruitment and exclusion of subject.

is a written test that consists of linking target as quickly as possible; integers in a numerical order in Part A (e.g., $1-2-3 \ldots 25)$, and alternating between numbers and letters in Part B (e.g., 1-A-2-B-3-C, etc.). The visual perception abilities, perceptual/motor speed and speed processing of subjects were assessed in Part A, and mental flexibility was assessed in Part B; vi) symbol Digit Modalities Test (SDMT) is used to assess processing speed (37). The score is the number of correct answers selected within $90 \mathrm{sec}$ and vii) Berg Balance Scale (BBS) is used to assess the balance control of the elderly persons (38). The highest score available is 56; a score of 40 indicated a falling risk.

Image acquisition. MRI scanning was performed on a Siemens Magnetom Trio 3.0T MRI System (Siemens AG, Erlangen, Germany) using a standard birdcage head transmit and receive coil at baseline and after the 3-month intervention. Functional images were acquired using a single-shot, gradient-recalled echo planar imaging sequence [repetition time $(\mathrm{TR})=2,000$ msec; echo time $(\mathrm{TE})=30 \mathrm{msec}$; flip angle $\left.(\mathrm{FA})=90^{\circ}\right]$. A total of 33 transverse slices [field of view $(\mathrm{FOV})=256 \times 256 \mathrm{~mm}^{2}$; in-plane matrix $=64 \times 64$; slice thickness $=4 \mathrm{~mm}$; inter-slice gap $=1 \mathrm{~mm}$; voxel size $=4 \times 4 \times 4 \mathrm{~mm}^{3}$ ] aligned along the anterior-posterior commissure line were acquired. For each subject, a total of 240 volumes were acquired, resulting in a total scan time of $480 \mathrm{sec}$. The subjects were instructed to simply rest with their eyes closed, to not think of anything in particular and to not fall asleep. The high-resolution 3D T1-weighted anatomical images were acquired in a sagittal orientation using a magnetization-prepared rapid gradient-echo sequence $\left(\mathrm{TR}=1,900 \mathrm{msec} ; \mathrm{TE}=2.52 \mathrm{msec} ; \mathrm{FA}=9^{\circ} ; \mathrm{FOV}=256 \times 256 \mathrm{~mm}^{2}\right.$; matrix size $=256 \times 256$; slice thickness $=1 \mathrm{~mm}$; inter-slice gap $=0.5 \mathrm{~mm}$; voxel size $=1 \times 1 \times 1 \mathrm{~mm}^{3} ; 176$ slices). Then, axial fluid-attenuated inversion recovery images (time of inversion=2,500 $\mathrm{msec}$; $\mathrm{TR}=9,000 \mathrm{msec} ; \mathrm{TE}=100 \mathrm{msec}$; slice thickness $=5 \mathrm{~mm}$ ) were obtained for diagnosis.

Image preprocessing and ALFF analysis. Functional images were preprocessed by one author using the Data Processing Assistant for Resting-State fMRI (DPARSF) (39). The steps were as follows: i) Digital Imaging and Communications in Medicine data was converted into Neuroimaging Informatics Technology Initiative data; ii) The first 10 images were removed; iii) slice timing correction was performed; iv) head motion correction was performed; v) The data was normalized (re-sliced at a resolution of $3 \times 3 \times 3 \mathrm{~mm}^{3}$ ); vi) spatial smoothing was performed ( $6 \mathrm{~mm}$ full-width-at-half-maximum isotropic Gaussian kernel); vii) filtering was performed $(0.01<\mathrm{f}<0.08 \mathrm{~Hz})$. Two subjects in the EG and three subjects in CG with head motions exceeding $2 \mathrm{~mm}$ of translation or 2 degrees of rotation throughout the course of the scan were excluded from the study.

Then the time series' were transformed to the frequency domain using a fast Fourier transform algorithm and the averaged square root of the spectrum across $0.01-0.08 \mathrm{~Hz}$ at each voxel was obtained as the ALFF. For standardizing variability among the subjects, mean ALFF was taken as the ALFF of each voxel divided by the mean of all voxels. The gray matter 
Table I. Baseline demographics and clinical characteristics.

\begin{tabular}{lccc}
\hline Characteristics & The exercise group $(\mathrm{n}=16)$ & The control group (n=16) & P-value \\
\hline Sex (male:female) & $5: 11$ & $4: 12$ & 0.70 \\
Age (years) & $70.6(6.2)$ & $69.1(8.1)$ & 0.58 \\
Education (years) & $10.4(2.6)$ & $9.7(2.7)$ & 0.47 \\
Mini-mental state examination & $27.3(1.3)$ & $27.1(1.2)$ & 0.68 \\
Montreal cognitive assessment & $22.6(2.1)$ & $22.9(1.7)$ & 0.65 \\
Wechsler memory scale-revised logical memory & $14.1(5.9)$ & $16.7(5.4)$ & 0.21 \\
Digit span test & $16.4(2.9)$ & $18.1(3.4)$ & 0.14 \\
Trail making test part A & $107.3(97.1)$ & $72.2(23.3)$ & 0.17 \\
Trail making test part B & $190.6(59.2)$ & $182.2(57.7)$ & 0.69 \\
Symbol digit modalities test & $31.8(9.6)$ & $34.1(10.9)$ & 0.53 \\
Berg balance scale & $55.1(1.4)$ & $55.4(1.0)$ & 0.56
\end{tabular}

Data are expressed as $\mathrm{n}$ or mean (standard deviation).

volumes were also obtained in the results preprocessed by DPARSF (39) (data not shown). The two-sample t-test, paired t-test and Pearson's correlation analysis were performed using the Resting-state fMRI Data Analysis Toolkit v1.8 (REST1.8) (http://www.restfmri.net) (40). The gray matter volume, age, gender, and education were added as covariates for excluding the related effects.

Statistical methods. Continuous variables are presented as mean (standard deviation). Sex distribution was assessed using a Chi-square test. The aforementioned statistical analyses were performed using SPSS version 16.0 software (SPSS, Inc., Chicago, IL, USA). A t-test was performed using REST1.8 (40) to determine the mean ALFF differences within the whole brain between the two groups (voxel-level $\mathrm{P}<0.05$, cluster size $>228$ voxels, corresponding to a corrected $\mathrm{P}<0.05$ as determined by AlphaSim) (http://afni.nimh.nih. gov/pub/dist/doc/manual/AlphaSim.pdf). Between-group comparisons were performed using two-sample t-tests. Within-group comparisons were performed using paired t-tests. $\mathrm{P}<0.05$ indicated that the difference between groups was statistically significant. A Pearson's correlation analysis was performed using REST1.8 (40) to determine the correlation between the ALFF and neuropsychological test scores (voxel-level $\mathrm{P}<0.05$; cluster size $>228$ voxels; corresponding to a corrected $\mathrm{P}<0.05$ as determined by AlphaSim).

\section{Results}

Baseline demographic and clinical characteristics. Overall, 32 subjects completed the whole study. The baseline demographics and clinical characteristics of the EG and CG are summarized in Table I. No significant differences in sex, age, education years or on MMSE, MoCA, WMS-R LM, DST, TMT-A, TMT-B, SDMT and BBS were identified between the two groups.

Neuropsychological measures of intervention effects. As demonstrated in Table II, within-group differences demonstrated that the scores of MMSE, MoCA, WMS-R LM and SDMT were significantly increased in the EG $(\mathrm{P}<0.05)$ compared with the baseline, but not in the CG. Between-group differences revealed that the change of WMS-R LM from baseline to 3 months in the EG was significantly higher than the change in the $\mathrm{CG}(\mathrm{P}<0.05)$.

$R S$-fMRI measures of intervention effects. No significant differences in the ALFF were identified between the two groups at the baseline (data not shown). Changes in the ALFF at the baseline and after the 3-month intervention were analyzed to evaluate the effect of the SDMIAD within each group. The EG demonstrated a significant increase in the ALFF in the bilateral fronto-temporal, entorhinal, anterior cingulate and parohippocampal cortex $(\mathrm{P}<0.05$; Fig. 2$)$. Whereas in the $\mathrm{CG}$, no significant differences in the aforementioned areas were identified between the pre- and post-intervention groups, however a small number areas of functional activation appeared in the right temporal and posterior cingulate cortex $(\mathrm{P}<0.05$; Fig. 3$)$. The location and volume of activated regions which exhibited significant changes (post-intervention -pre-intervention) in the EG or the CG are presented in Table III.

Correlation between ALFF and neuropsychological test scores. No significant correlation was identified between the ALFF and neuropsychological test scores.

\section{Discussion}

In the current study, the ALFF was used to detect abnormal brain activation after a 3-month SDMIAD intervention in older adults with MCI. It was demonstrated that the EG exhibited an increased ALFF in several areas, including the bilateral fronto-temporal, entorhinal, anterior cingulate and parohippocampal cortex following the intervention. Whereas the CG only demonstrated increases in the ALFF in a small number of areas; including the right temporal and posterior cingulate cortex. Following the intervention, neuropsychological tests determined that MMSE, MoCA, WMS-R LM and SDMT improved in the EG, but not in the CG.

In patients with $\mathrm{AD}$, the earliest occurrence of pathological damage was demonstrated to be in the entorhinal cortex; the 


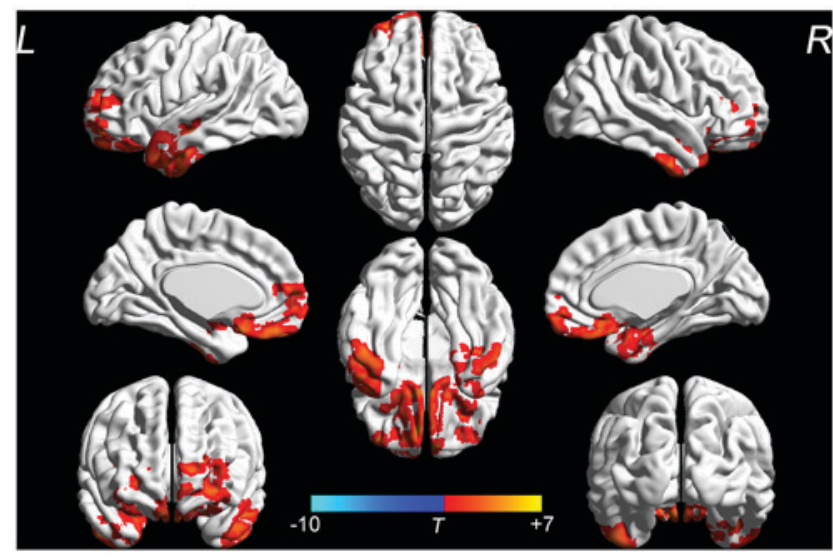

Figure 2. ALFF results of brain regions demonstrate significant differences in the exercise group pre- and post-intervention. The $3 \mathrm{D}$ pseudo-color map revealed increases in ALFF following the intervention (red). Results are displayed at $\mathrm{P}<0.05$ corrected by AlphaSim. ALFF, amplitude of low-frequency fluctuation; $\mathrm{L}$, left; $\mathrm{R}$, right. $\mathrm{T}$, $\mathrm{T}$ value obtained from paired t-test of the exercise group.

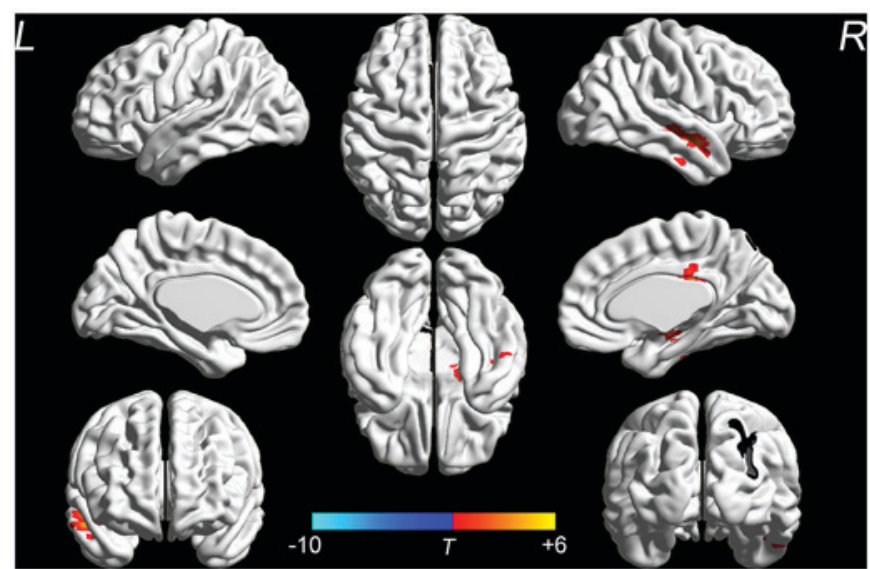

Figure 3. ALFF results of brain regions demonstrate significant differences in the control group pre- and post-intervention. The 3D pseudo-color map revealed increases in ALFF following the intervention (red). Results are displayed at $\mathrm{P}<0.05$ corrected by AlphaSim. ALFF, amplitude of low-frequency fluctuation; $\mathrm{L}$, left; $\mathrm{R}$, right. $\mathrm{T}, \mathrm{T}$ value obtained from paired t-test of the control group.

damage then extends to the hippocampus and other limbic structures, and finally occurs in the neocortical region (41). Celone et al (42) indicated that activation in the hippocampus and lateral parietal lobe of patients with MCI increased compared with normal elderly subjects using RS-fMRI. Han et al (43) revealed changes in ALFF were abnormal in multiple brain regions in MCI. In the current study, as ALFF was increase in several regions, the SDMIAD may be effective in the treatment of patients with AD or MCI.

Impaired memory function is the first and the most common observable symptom of MCI (44). The aforementioned study also demonstrated that olfactory function was impaired in the early stages of the condition $(45,46)$. Wilson et al (47) demonstrated that olfactory dysfunction in the elderly was likely to develop into MCI. Many important cognitive functions depend on the frontal cortex and medial temporal lobe, including memory, executive control and 
Table III. Location and spatial extent of activated regions post and pre-intervention in EG and CG.

\begin{tabular}{|c|c|c|c|c|c|c|}
\hline \multirow[b]{2}{*}{ Clusters } & \multirow[b]{2}{*}{ Brodmann area } & \multirow[b]{2}{*}{ Voxels } & \multicolumn{3}{|c|}{ MNI coordinates (mm) } & \multirow[b]{2}{*}{ t-value } \\
\hline & & & $\mathrm{X}$ & $\mathrm{Y}$ & $\mathrm{Z}$ & \\
\hline \multicolumn{7}{|l|}{ EG } \\
\hline Left inferior temporal gyrus & 20 & & & & & \\
\hline Left middle temporal gyrus & 21 & 487 & -57 & 0 & -24 & 5.33 \\
\hline Left superior temporal gyrus & 22 & & & & & \\
\hline Left dorsal entorhinal cortex & 34 & & & & & \\
\hline Left retrosubicular area & 48 & & & & & \\
\hline Right inferior temporal gyrus & 20 & & & & & \\
\hline Right ventral entorhinal cortex & 28 & 283 & 30 & 6 & -36 & 5.29 \\
\hline Right parahippocampal gyrus & 36 & & & & & \\
\hline Left anterior prefrontal cortex & 10 & & & & & \\
\hline Bilateral orbitofrontal area & 11 & 782 & -9 & 48 & -24 & 7.34 \\
\hline Left dorsal anterior cingulate cortex & 32 & & & & & \\
\hline Bilateral subgenual area & 25 & & & & & \\
\hline Right orbitofrontal area & 11 & & & & & \\
\hline Right pars orbitalis & 47 & 279 & 30 & 36 & 18 & 4.06 \\
\hline Right retrosubicular area & 48 & & & & & \\
\hline \multicolumn{7}{|l|}{ CG } \\
\hline Right inferior temporal gyrus & 20 & & & & & \\
\hline Right middle temporal gyrus & 21 & & & & & \\
\hline Right superior temporal gyrus & 22 & 310 & 6 & -30 & 30 & 5.83 \\
\hline Right ventral posterior cingulate cortex & 23 & & & & & \\
\hline Right retrosubicular area & 48 & & & & & \\
\hline
\end{tabular}

EG, the exercise group; CG, the control group.

learning (48-50). The medial temporal lobe is the structural basis of memory. The frontal cortex is closely associated with human mental activity, especially the emotion functioning and cognitive function. Physical activity has been associated with sparing of volume of the prefrontal and temporal brain regions, which reduces the risk for cognitive impairment (51). Li et al (19) indicated that exercise intervention would improve the resting-state functional connectivity between the medial prefrontal cortex and medial temporal lobe. Bilateral superior and middle frontal gyri are associated with selective attention dysfunction (52). The cingulate gyrus is an important part of the limbic system, which is the core of the emotional loop (53). Anterior cingulate cortex and frontal lobe brain areas are mainly responsible for attention and executive function (52). In the current study, the increased functional activation of the bilateral fronto-temporal, entorhinal, anterior cingulate and parohippocampal cortex indicated that the 3-month SDMIAD intervention can effectively improve cognitive functions, including memory and executive function, and potentially olfactory function, in patients with MCI. These neuropsychological results were similar to those of the RS-fMRI. A statistical test demonstrated that no significant correlation was identified between the ALFF and neuropsychological test scores. This may be due to the relatively small sample size.
In contrast, no significant improvement in the neuropsychological tests were identified between the baseline and 3-month results in the CG. According to RS-fMRI, the CG had an increased ALFF in a small number of areas, including the right temporal and posterior cingulated cortex, which were different from the areas of increased function in the EG. These results may indicate that older adults with MCI in the CG counteract cognition decline via the increased functional activation of a small number of areas, but it cannot improve cognitive function. Conversely, the results from the EG demonstrated that the SDMIAD provided an effective way to compensate for MCI or AD. Cabeza (54) proposed that the prefrontal and medial temporal cortexes are particularly vulnerable to the effects of aging, and that age-related neural decline is asymmetric as the right hemisphere is more vulnerable to aging than the left. Subsequently, Cabeza et al (55) suggested that there was a decline in right fronto-temporal function in older people with memory impairment and that there was a lack of compensation in the left cerebral hemisphere; however those who had a good memory counteracted age-related neural decline in the right hemisphere via left hemisphere compensation, thus maintaining a normal memory. The current study revealed that the functional activation of bilateral fronto-temporal cortex was increased significantly after 3-month SDMIAD intervention, whereas only a small number of areas of functional activation appeared in the right 
temporal cortex in the $\mathrm{CG}$, which lacked compensation in the left cerebral hemisphere.

The present study had several limitations. Firstly, the sample size was small, which may have reduced the statistical power of the analysis and led to false negative findings, such as the lack of the correlation between the ALFF and neuropsychological test scores. In future studies by our group, the sample sizes will be larger with the hope of confirming the correlation and thus the intervention effects. Secondly, the long-term effects of the intervention were not assessed in a follow-up. Further studies are needed to examine whether intervention-induced brain changes are maintained over time. Thirdly, only the effect of one intervention (aerobic dance) of aerobic exercise at one levels of intensity (moderate) was assessed. Future studies will evaluate the intervention effects of different forms and intensities on cognitive function.

In conclusion, the ALFF changes observed in RS-fMRI provide evidence that the SDMIAD can significantly increase the functional activation of several brain regions, and help to effectively improve the cognitive function in older adults with MCI. RS-fMRI provides a quantitative method for evaluating the effect of aerobic exercise on cognitive function.

\section{Acknowledgements}

Not applicable.

\section{Funding}

The current study was supported by the Science and Technology Department of Jiangsu Province (grant no. 2013-DB13).

\section{Availability of data and materials}

The datasets used and/or analyzed during the current study are available from the corresponding author on reasonable request.

\section{Authors' contributions}

JW conceived the current study. MQ performed the data analyses and wrote the manuscript. YZ interpreted the patient data and wrote the manuscript. LZ examined and analyzed the data. TW recruited patients and performed neuropsychological tests. All authors read and approved the final manuscript.

\section{Ethics approval and consent to participate}

The current study was approved by the Ethics Committee of the First Affiliated Hospital of Nanjing Medical University (Nanjing, China). All participants provided written informed consent prior to participating in the current study.

\section{Patient consent for publication}

Not applicable.

\section{Competing interests}

The authors declare that they have no competing interests.

\section{References}

1. Sarazin M, de Souza LC, Lehéricy S and Dubois B: Clinical and research diagnostic criteria for Alzheimer's disease. Neuroimaging Clin N Am 22: 23-32,viii, 2012.

2. Albert MS, DeKosky ST, Dickson D, Dubois B, Feldman HH, Fox NC, Gamst A, Holtzman DM, Jagust WJ, Petersen RC, et al: The diagnosis of mild cognitive impairment due to Alzheimer's disease: Recommendations from the National Institute on Aging-Alzheimer's Association workgroups on diagnostic guidelines for Alzheimer's disease. Alzheimers Dement 7: 270-279, 2011.

3. Inouye K, Pedrazzani ES and Pavarini SC: Alzheimer's disease influence on the perception of quality of life from the elderly people. Rev Esc Enferm USP 44: 1093-1099, 2010 (In Portuguese).

4. Barnes DE and Yaffe K: The projected effect of risk factor reduction on Alzheimer's disease prevalence. Lancet Neurol 10: 819-828, 2011.

5. Astell-Burt T and Feng $\mathrm{X}$ : Is the risk of developing Alzheimer's disease really higher in rural areas? A multilevel longitudinal study of 261,669 Australians aged 45 years and older tracked over 11 years. Health Place 54: 132-137, 2018.

6. Rasmussen KL, Tybjærg-Hansen A, Nordestgaard BG and Frikke-Schmidt R: Absolute 10-year risk of dementia by age, sex and APOE genotype: A population-based cohort study. CMAJ 190: E1033-E1041, 2018.

7. Sofi F, Valecchi D, Bacci D, Abbate R, Gensini GF, Casini A and Macchi C: Physical activity and risk of cognitive decline: A meta-analysis of prospective studies. J Intern Med 269: 107-117, 2011.

8. Lautenschlager NT, Cox K and Kurz AF: Physical activity and mild cognitive impairment and Alzheimer's disease. Curr Neurol Neurosci Rep 10: 352-358, 2010.

9. Gallaway PJ,Miyake H, Buchowski MS, Shimada M, Yoshitake Y, Kim AS and Hongu N: Physical activity: A viable way to reduce the risks of mild cognitive impairment, Alzheimer's disease and vascular dementia in older adults. Brain Sci 7: pii: E22, 2017.

10. Jones LW, Douglas PS, Khouri MG, Mackey JR, Wojdyla D, Kraus WE, Whellan DJ and O'Connor CM: Safety and efficacy of aerobic training in patients with cancer who have heart failure: An analysis of the HF-ACTION randomized trial. J Clin Oncol 32: 2496-2502, 2014

11. Brown BM, Peiffer JJ, Sohrabi HR, Mondal A, Gupta VB, Rainey-Smith SR, Taddei K, Burnham S, Ellis KA, Szoeke C, et al: Intense physical activity is associated with cognitive performance in the elderly. Transl Psychiatry 2: e191, 2012.

12. Kerr J, Marshall SJ, Patterson RE, Marinac CR, Natarajan L, Rosenberg D, Wasilenko K and Crist K: Objectively measured physical activity is related to cognitive function in older adults. J Am Geriatr Soc 61: 1927-1931, 2013.

13. Doi T, Makizako H, Shimada H, Tsutsumimoto K, Hotta R, Nakakubo S, Park H and Suzuki T: Objectively measured physical activity, brain atrophy, and white matter lesions in older adults with mild cognitive impairment. Exp Gerontol 62: 1-6, 2015.

14. Zhang S, Hu Q, Tang T, Liu C, Li C, Zang YY and Cai WX: Changes in gray matter density, regional homogeneity, and functional connectivity in methamphetamine-associated psychosis: A resting-state functional magnetic resonance imaging (fMRI) study. Med Sci Monit 24: 4020-4030, 2018.

15. Biswal B,Zerrin Yetkin F, Haughton VM and Hyde JS: Functional connectivity in the motor cortex of resting human brain using echo-planar MRI. Magn Reson Med 34: 537-541, 1995.

16. Zang YF, He Y, Zhu CZ, Cao QJ, Sui MQ, Liang M, Tian LX, Jiang TZ and Wang YF: Altered baseline brain activity in children with ADHD revealed by resting-state functional MRI Brain Dev 29: 83-91, 2007.

17. Zou QH, Zhu CZ, Yang Y, Zuo XN, Long XY, Cao QJ, Wang YF and Zang YF: An improved approach to detection of amplitude of low-frequency fluctuation (ALFF) for resting-state fMRI: Fractional ALFF. J Neurosci Methods 172: 137-141, 2008.

18. Colcombe SJ, Erickson KI, Scalf PE, Kim JS, Prakash R, McAuley E, Elavsky S, Marquez DX, Hu L and Kramer AF: Aerobic exercise training increases brain volume in aging humans. J Gerontol A Biol Sci Med Sci 61: 1166-1170, 2006.

19. Li R, Zhu X, Yin S, Niu Y, Zheng Z, Huang X, Wang B and Li J: Multimodal intervention in older adults improves resting-state functional connectivity between the medial prefrontal cortex and medial temporal lobe. Front Aging Neurosci 6: 39, 2014. 
20. Colcombe SJ, Kramer AF, Erickson KI, Scalf P, McAuley E, Cohen NJ, Webb A, Jerome GJ, Marquez DX and Elavsky S: Cardiovascular fitness, cortical plasticity, and aging. Proc Natl Acad Sci USA 101: 3316-3321, 2004.

21. Rosano C, Venkatraman VK, Guralnik J, Newman AB Glynn NW, Launer L, Taylor CA, Williamson J, Studenski S Pahor $\mathrm{M}$ and Aizenstein H: Psychomotor speed and functional brain MRI 2 years after completing a physical activity treatment. J Gerontol A Biol Sci Med Sci 65: 639-647, 2010.

22. Xi Q, Zhao XH, Wang PJ, Guo QH and He Y: Abnormal intrinsic brain activity in amnestic mild cognitive impairment revealed by amplitude of low-frequency fluctuation: A resting-state functional magnetic resonance imaging study. Chin Med J (Engl) 126 2912-2917, 2013

23. Xi Q, Zhao XH, Wang PJ, Guo QH, Yan CG and He Y: Functional MRI study of mild Alzheimer's disease using amplitude of low frequency fluctuation analysis. Chin Med J (Engl) 125: 858-862, 2012

24. Xi Q, Zhao X, Wang P, Guo Q, Jiang H, Cao X, He Y and Yan C: Spontaneous brain activity in mild cognitive impairment revealed by amplitude of low-frequency fluctuation analysis: A resting-state fMRI study. Radiol Med 117: 865-871, 2012.

25. Petersen RC, Caracciolo B, Brayne C, Gauthier S, Jelic V and Fratiglioni L: Mild cognitive impairment: A concept in evolution. J Intern Med 275: 214-228, 2014.

26. Zhang H, Zhao L, Yang S, Chen Z, Li Y, Peng X, Yang Y and Zhu M: Clinical observation on effect of scalp electroacupuncture for mild cognitive impairment. J Tradit Chin Med 33: 46-50, 2013.

27. Folstein MF, Folstein SE and McHugh PR: 'Mini-mental state'. A practical method for grading the cognitive state of patients for the clinician. J Psychiatr Res 12: 189-198, 1975.

28. Yu J, Li J and Huang X: The Beijing version of the Montreal Cognitive Assessment as a brief screening tool for mild cognitive impairment: A community-based study. BMC Psychiatry 12 : $156,2012$.

29. Ryu HG, Youn SW and Kwon OD: Lack of association between apolipoprotein $\mathrm{E}$ polymorphism with age at onset of subcortical vascular dementia. Dement Geriatr Cogn Disord Extra 2: 1-9, 2012.

30. American College of Sports Medicine Position Stand. The recommended quantity and quality of exercise for developing and maintaining cardiorespiratory and muscular fitness and flexibility in healthy adults. Med Sci Sports Exerc 30: 975-991, 1998

31. Okin PM, Ameisen $\mathrm{O}$ and Kligfield P: A modified treadmill exercise protocol for computer-assisted analysis of the ST segment/heart rate slope: Methods and reproducibility. J Electrocardiol 19: 311-318, 1986

32. Luis CA, Keegan AP and Mullan M: Cross validation of the montreal cognitive assessment in community dwelling older adults residing in the Southeastern US. Int J Geriatr Psychiatry 24: 197-201, 2009.

33. Nasreddine ZS, Phillips NA, Bédirian V, Charbonneau S, Whitehead V, Collin I, Cummings JL and Chertkow H: The montreal cognitive assessment, MoCA: A brief screening tool for mild cognitive impairment. J Am Geriatr Soc 53: 695-699, 2005.

34. Wang J, Zou YZ, Cui JF, Fan HZ, Chen R, Chen N, Yao J, Duan JH, Yan LJ, He XL and Jiang X: Revision of the wechsler memory scale-Fourth edition of Chinese version (adult battery). Chin Ment Health J 29: 53-59, 2015.

35. Lü J, Sun M, Liang L, Feng Y, Pan X and Liu Y: Effects of momentum-based dumbbell training on cognitive function in older adults with mild cognitive impairment: A pilot randomized controlled trial. Clin Interv Aging 11: 9-16, 2015.

36. Perrochon A and Kemoun G: The walking trail-making test is an early detection tool for mild cognitive impairment. Clin Interv Aging 9: 111-119, 2014

37. Cherbuin N, Sachdev P and Anstey KJ: Neuropsychological predictors of transition from healthy cognitive aging to mild cognitive impairment: The PATH through life study. Am J Geriatr Psychiatry 18: 723-733, 2010.
38. Telenius EW, Engedal $\mathrm{K}$ and Bergland $\mathrm{A}$ : Inter-rater reliability of the berg balance scale, $30 \mathrm{~s}$ chair stand test and $6 \mathrm{~m}$ walking test, and construct validity of the berg balance scale in nursing home residents with mild-to-moderate dementia. BMJ Open 5: e008321, 2015.

39. Chao-Gan Y and Yu-Feng Z: DPARSF: A MATLAB toolbox for 'Pipeline' data analysis of resting-state fMRI. Front Syst Neurosci 4: 13, 2010.

40. Song XW, Dong ZY, Long XY, Li SF, Zuo XN, Zhu CZ, He Y, Yan CG and Zang YF: REST: A toolkit for resting-state functional magnetic resonance imaging data processing. PLoS One 6: e25031, 2011

41. Kordower JH, Chu Y, Stebbins GT, DeKosky ST, Cochran EJ, Bennett D and Mufson EJ: Loss and atrophy of layer II entorhinal cortex neurons in elderly people with mild cognitive impairment. Ann Neurol 49: 202-213, 2010.

42. Celone KA, Calhoun VD, Dickerson BC, Atri A, Chua EF, Miller SL, DePeau K, Rentz DM, Selkoe DJ, Blacker D, et al: Alterations in memory networks in mild cognitive impairment and Alzheimer's disease: An independent component analysis. J Neurosci 26: 10222-10231, 2006.

43. Han Y, Wang J, Zhao Z, Min B, Lu J, Li K, He Y and Jia J: Frequency-dependent changes in the amplitude of low-frequency fluctuations in amnestic mild cognitive impairment: A resting-state fMRI study. Neuroimage 55: 287-295, 2011.

44. Jonker C, Geerlings MI and Schmand B: Are memory complaints predictive for dementia? A review of clinical and population-based studies. Int J Geriatr Psychiatry 15: 983-991, 2000.

45. Doty RL: The olfactory vector hypothesis of neurodegenerative disease: Is it viable? Ann Neurol 63: 7-15, 2008.

46. Doty RL: The olfactory system and its disorders. Semin Neurol 29: 74-81, 2009

47. Wilson RS, Schneider JA, Arnold SE, Tang Y, Boyle PA and Bennett DA: Olfactory identification and incidence of mild cognitive impairment in older age. Arch Gen Psychiatry 64: 802-808, 2007.

48. Rhodes MG: Age-related differences in performance on the Wisconsin card sorting test: A meta-analytic review. Psychol Aging 19: 482-494, 2004.

49. Burke SN and Barnes CA: Neural plasticity in the ageing brain. Nat Rev Neurosci 7: 30-40, 2006.

50. Cabeza R and Dennis NA: Frontal lobes and aging: Deterioration and compensation. In: Principles of Frontal Lobe Function. Stuss DT and Knight RT (eds). Oxford University Press, Oxford, United Kingdom, pp628-652, 2013.

51. Erickson KI, Raji CA, Lopez OL, Becker JT, Rosano C, Newman AB, Gach HM, Thompson PM, Ho AJ and Kuller LH: Physical activity predicts gray matter volume in late adulthood: The Cardiovascular Health Study. Neurology 75: 1415-1422, 2010.

52. Smith EE and Jonides J: Storage and executive processes in the frontal lobes. Science 283: 1657-1661, 1999.

53. Hadland KA, Rushworth MF, Gaffan D and Passingham RE: The effect of cingulate lesions on social behaviour and motion. Neuropsychologia 41: 919-931, 2003.

54. Cabeza R: Hemispheric asymmetry reduction in older adults: The HAROLD model. Psychol Aging 17: 85-100, 2002.

55. Cabeza R, Anderson ND, Locantore JK and McIntosh AR: Aging gracefully: Compensatory brain activity in high-performing older adults. Neuroimage 17: 1394-1402, 2002.

This work is licensed under a Creative Commons Attribution-NonCommercial-NoDerivatives 4.0 International (CC BY-NC-ND 4.0) License. 